\title{
An Application of the Inverse Method in the Vibroacoustic Analysis of Industrial Rooms
}

\author{
J. PIECHOWICZ*
}

\author{
AGH University of Science and Technology, Department of Mechanics and Vibroacoustics \\ al. A. Mickiewicza 30, 30-059 Krakow, Poland
}

\begin{abstract}
The problem of identification and localization of sound sources inside industrial rooms, including cases when the density of sound sources is considerable, can be solved by application of inverse methods. The experimental knowledge about the distribution of acoustic field in the neighborhood of the examined object, followed by the reversal of the model used for sound generation and propagation, leads to evaluation of the object's acoustic power. For the cases when determination of the rooms' acoustical properties is required the combination of the inverse method with the boundary elements method allows the calculation of acoustic impedance for the surface delimiting the examined room. The paper presents the prospects of the inverse method application in the mentioned vibroacoustic problems.
\end{abstract}

PACS: 43.20.Ye, 43.55.Ev, 43.55.Dt

\section{Introduction}

The application of inverse methods in combination with other computer methods, like the finite and boundary element methods or acoustic methods for determination of acoustic signal propagation in enclosed rooms, may become a more effective way for localization and identification of sound sources. It also allows the reconstruction of compound sound sources [1] and indication of the areas on the machinery body or its functional nodes which are exceedingly vibroactive [2]. The inverse methods can be also applied in determination of acoustic parameters of an industrial room, such as the values of sound absorption coefficient or the acoustic impedance of the walls $[3,4]$.

For prediction of the distribution of acoustic field parameters in industrial rooms dedicated software packages are used, based on the geometrical acoustics methods [5]. The room interior is modeled and the picture of acoustic field distribution within the volume of the industrial room is produced. For most software packages the boundary conditions are defined by the sound absorption coefficient for the material applied on the surface delimiting the room. Calculational problems are encountered e.g. for low frequency waves, when the wavelength is comparable with the dimensions of the reflecting surface. These problems of the acoustic field analysis for industrial rooms can be effectively solved by application of other numerical methods like finite element method

* e-mail: piechowi@agh.edu.pl
(FEM) or boundary element method (BEM) [6, 7]. In such cases the proper definition of the boundary conditions is of fundamental importance. The properties of the room's volume boundary can be defined by specification of the surface acoustic impedance or acoustic admittance in the complex form. The paper presents the prospects of inverse method application in the vibroacoustic studies of industrial rooms with examples from experimental studies conducted by the author.

\section{The inverse method for determining the acoustic power of noise sources}

Modeling of real sound sources by application of substitute sources (representing functional elements of the machinery like engine, fan, cogwheels etc.) is related to the indication of their location in the interaction space and specification of the directional characteristics of the sound radiation. The machinery emits sound producing the acoustic pressure registered by a number of microphones distributed in the surrounding space. From the acoustic measurements the distributions of values of acoustic field parameters (e.g. acoustic pressure) can be determined in many reception points. By inverting the modeled sound propagation path from the source to the observation point one can determine the acoustic power of the sound source (Fig. 1). When applying the inverse method one replaces the actual sound source by a system of substitute sources, and then simulates the acoustic parameters of these sources in order to obtain the distribution of the acoustic field produced by the system as close as possible to the distribution of the acoustic field produced around the actual sound source $[8,9]$. 


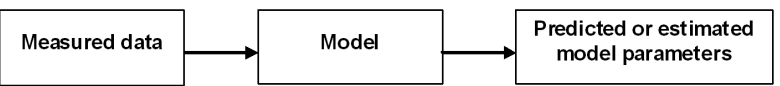

Fig. 1. Inverse modeling.

Modeling the vibroacoustic process of sound radiation by the source, one can write down that the values of acoustic pressure in the observation points will be determined from the following formula [1]:

$$
\hat{\boldsymbol{p}}=\boldsymbol{G} \cdot \boldsymbol{q}+\boldsymbol{e},
$$

where $\boldsymbol{G}-m \times n$ matrix, representing the compound frequency response function, connecting the acoustic pressure values distribution model with the substitute model, $m$ - number of observation points, $n$ - number of sound source parameters, $\boldsymbol{p}$ is the $m$-dimensional vector of the measured amplitudes of acoustic pressure in the observation points, $\boldsymbol{q}$ is the $n$-dimensional vector of complex values for the sound source parameters of the model, $e=\hat{p}-p$ is the error vector, defined as the difference between the acoustic pressure determined from the noise propagation model and the value measured in a given observation point.

During the search for solution of the problem it is assumed that the locations of the noise sources in the examined machinery are known and the acoustic pressure values in a finite number $m$ of observation point locations are measured. In order to minimize the error vector the acoustic parameters of individual sound sources of the model are optimized. The methods usually applied are the least distance or least squares methods or the singular value decomposition (SVD). The vector of estimated values for parameters of individual sources (e.g. acoustic power) $\boldsymbol{N}$ is determined from the following formula [1]:

$$
\boldsymbol{N}=\boldsymbol{G}^{+} \hat{\boldsymbol{p}},
$$

in which $\boldsymbol{G}^{+}$is a pseudo-inverse matrix, defined by the formula:

$$
\boldsymbol{G}^{+}=\left(\boldsymbol{G}^{\mathrm{H}} \boldsymbol{G}\right)^{-1} \boldsymbol{G}^{\mathrm{H}},
$$

where $\boldsymbol{G}^{+}=\left[\boldsymbol{G}^{\mathrm{H}} \boldsymbol{G}\right]^{-1}$ matrix, is a pseudo-inverse of the $\boldsymbol{G}$ matrix, which reduces to $\boldsymbol{G}^{+}=\boldsymbol{G}^{-}$when $M=N$, i.e. when the $\boldsymbol{G}$ matrix is a square matrix. Superscript $\mathrm{H}$ denotes Hermitian transpose.

The $\boldsymbol{G}^{+}$matrix can be determined using the SVD method

$$
\boldsymbol{G}^{+}=\boldsymbol{V} \boldsymbol{\Sigma}^{+} \boldsymbol{U}^{\mathrm{H}},
$$

where $\boldsymbol{U}, \boldsymbol{V}$ - orthogonal matrices, $\boldsymbol{\Sigma}^{+}$- the pseudo-inverse of $\Sigma$ with the individual elements of the matrix generated by $\boldsymbol{\Sigma}^{+}=\left[s^{-1} \mid 0\right], s^{-1}=\operatorname{diag}\left(1 / \sigma_{1, \ldots}, 1 / \sigma_{N}\right)$.

A good measure of accuracy of the performed simulations is a value of the $\kappa(G)$ expression [10]:

$$
\kappa(\boldsymbol{G})=\frac{\sigma_{\max }}{\sigma_{\min }},
$$

where $\sigma_{\max }, \sigma_{\min }$ are respectively, the maximum and minimum singular values of $G$.
A large ratio of maximum to minimum singular value of $G$ will significantly amplify small measurement disturbances in $\boldsymbol{p}$. Relative error of the estimate $\delta e$ during the determination of model parameters for the $m$-th term of $e$ :

$$
\begin{aligned}
& \boldsymbol{e}=\left[e_{m}\right], \quad \boldsymbol{p}=\left[p_{m}\right] \text { where } m=1, \ldots, M, \\
& \left|\frac{\sigma e_{m}}{e_{m}}\right| \leq \kappa(\boldsymbol{G})\left|\frac{\delta p_{m}}{p_{m}}\right| .
\end{aligned}
$$

The application of inverse methods is related to reconstruction of solutions by using certain functionals, determined on the sets of solutions or reconstruction of operators describing the problems. Such an inversion problem is incorrectly formulated in the Hadamard sense, which means that small changes of the considered functionals may lead to quite considerable changes in the solutions. If additional restrictions are imposed on the set of acceptable solutions, then stable solutions can be obtained, that are not sensitive to the changes of data, or to put it in other words the problems are well-conditioned. The approximate solutions can be discussed on the basis of approximate data, by applying various regularization methods [10-13]. Inverse determination of sound power levels of individual machines - on the basis of sound pressure measurements in the observation points - is very sensitive to disturbances in receiving points. The accuracy of sound power reconstruction is largely determined by the conditioning of the matrix to be inverted. The Tikhonov regularization [14] improves the accuracy of acoustic source strength of the model reconstructed poorly by the least-squares method alone. In applying the Tikhonov regularization, we have to consider two factors: the manner by which the result should be regularized and the amount of regularization. The method of regularization is determined by the choice of regularization operator.

The possibility to apply the inverse methods in acoustics is conditioned by the collection of data from a sufficient number of reception points located in the acoustic field surrounding the examined object [14]. For both cases, namely the determination of acoustic parameters of a machinery or the acoustic parameters of the room itself, multi-channel data acquisition system has been used, based on the National Instruments PXI-1042Q device, with three 8-channel NI-PXI-4472B data input cards, allowing simultaneous 24 channel registration of signals coming from 1/4" GRAS 40PQ and Bruel\&Kjaer 4958 matrix microphones. The measurement procedure has been executed by dedicated software packages elaborated in the LabView environment.

Verification of the application of the acoustic power determination method has been carried out for many machinery setups and industrial devices, e.g. such a study has been carried out for a series of $\mathrm{AC}$ power generators with the output power ranging from $800 \mathrm{~W}$ to $3500 \mathrm{~W}$. Sound pressure level and shift phase angle of signals were recorded by microphones placed around machines. The analysis was carried out with the frequency resolution 
$10 \mathrm{~Hz}$ and in $1 / 3$ octave bands frequencies. Applying the formula (2) we obtain the acoustic power of the noise sources. The results are presented in Fig. $2 \mathrm{a}$ and $\mathrm{b}-$ the determined sound power spectra for the $3500 \mathrm{~W}$ electric power generator are shown for various external load values. The sound power levels of each machine were determined by the algorithm described above. It is important that the underlying model calculations (formulae (1) and (2)) are prepared once for the entire type series of measurements. This only requires amendments to the changing dimensions of the generators in series.

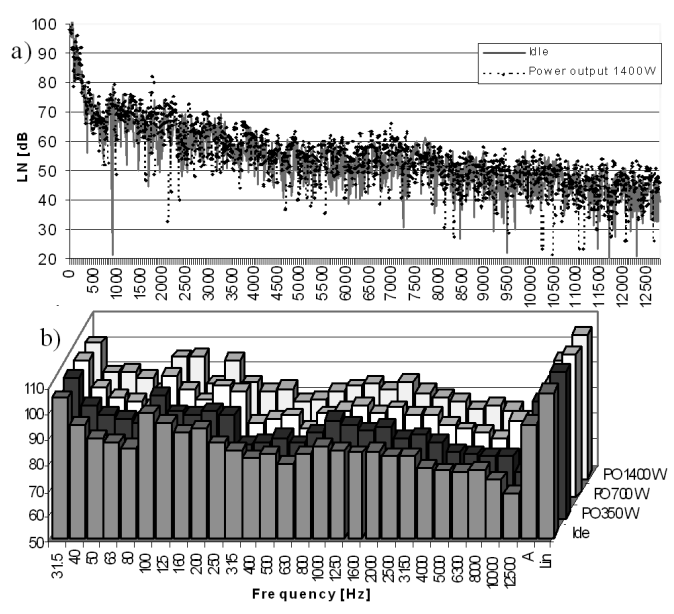

Fig. 2. Sound power spectra of $3500 \mathrm{~W}$ electric power generator determined using inverse methods: (a) frequency spectrum, (b) 1/3 octave sound power spectrum.

\section{Estimation of acoustic impedance of the walls based on an inverse method}

Knowing the acoustic power of the sound source one can model the distributions of the acoustic field parameters in the industrial room. For the needs of acoustic field modeling in volumes that are big in the wavelength scale several dedicated computer programs are available, while for the small rooms, the dimensions of which are comparable to the wavelength of the propagating acoustic wave, the methods of wave acoustics, e.g. boundary or finite element methods are much more accurate. Discussing the problems of acoustic field distribution [15], that is produced in a given room by a single acoustic source of a known acoustic power, using the boundary element method one can obtain the information regarding the approximate values of acoustic pressure that can be expected at the observation points during the actual measurement. The applicability (correctness) of the respective numerical model for the BEM software provides a basis for formulation of the inverse problem - the determination of the acoustic impedance $Z_{W}$ for the walls of the room from the known distribution of the acoustic pressure values within the room. The acoustic impedance $Z_{W x}$ in a given point $x$ at the surface of the wall $W$ can be defined as:

$$
Z_{W x}=\frac{p_{W x}}{v_{W x}}
$$

where $p_{W x}$ is the acoustic pressure at the point $x, v_{W x}$ is the acoustic particle velocity at the point $x$.

In most typical application, the propagation of acoustic waves are well modeled by the Helmholtz equation

$$
\nabla^{2} p+k^{2} p=0
$$

where $k=\omega / c$ is the wave number which defines relationship between the angular frequency $\omega$ and velocity of propagation $c$ of the wave.

The boundary conditions are typically either the pressure or acoustic velocity. The acoustic pressure in Eq. (7) is a function of position only and the derivative of pressure is related to the acoustic velocity in the form

$$
\frac{\partial p}{\partial n}=-j \omega \rho_{0} v_{n}
$$

where $\rho_{0}$ is the medium density in the room.

The dependence between the acoustic pressure $p_{R}$, measured in an arbitrary point within the room, and the acoustic pressure $p_{W}$ and the velocity of the acoustic particle $v_{W}$ at the boundary of the area, in the stationary acoustic field conditions is expressed by the HelmholtzKirchhoff equation [6]:

$$
\oint_{S}\left(p_{W} \frac{\partial G(r)}{\partial n}+j \omega \rho G(r) v_{W}\right) \mathrm{d} S+p_{R}=0,
$$

where $G(r)$ is the Green function $G=\frac{\mathrm{e}^{-\mathrm{j} k r}}{4 \pi r}, r=$ $\left|r_{R}-r_{W}\right|$ - distance between the point at the wall surface of the room and the reception point located inside the room.

The surface area delimiting the room in the model is divided into $N$ equivalent elements $W_{i}$ and the values of acoustic pressure are measured in $M$ reception points within the room. The model discretization using the boundary element method leads to the following matrix equation [3]:

$$
\sum_{k=1}^{N} p_{W k} h_{i k}-\sum_{k=1}^{N} v_{W k} l_{i k}=-p_{R}
$$

where $i=1,2,3 \ldots M$.

The $h_{i k}$ and $l_{i k}$ coefficients are defined as:

$$
\begin{aligned}
& h_{i, l}=\int_{s_{l}} \frac{G(r)}{\partial n} \mathrm{~d} s, \\
& l_{i, l}=-\mathrm{j} \rho \omega \int_{s_{l}} G(r) \mathrm{d} s,
\end{aligned}
$$

where $i=1,2, \ldots M ; k=1,2, \ldots, N$.

Therefore in the matrix form Eq. (10) can be written as

$$
\boldsymbol{H}_{R} \boldsymbol{p}_{W}-\boldsymbol{L}_{R} \boldsymbol{v}_{W}=-\boldsymbol{p}_{R}
$$

where $\boldsymbol{p}_{W}$ is the acoustic pressure at the wall surface (parameter of the volume boundary), the $\boldsymbol{H}_{R}$ and $\boldsymbol{L}_{R}$ matrices present the dependences between the point at the wall surface and the acoustic pressure measurement 
point located inside the room. If the points selected for the measurement of the acoustic pressure are located at the wall surface, and additionally assuming that the node points are located at the smooth surface of the wall $W$, Eq. (11) will take the form

$$
\boldsymbol{H}_{W} \boldsymbol{p}_{W}-\boldsymbol{L}_{W} \boldsymbol{v}_{W}=0,
$$

where $\boldsymbol{H}_{W}$ and $\boldsymbol{L}_{W}$ matrices of mutual effect coefficients between the points at the boundary surface $W$. Therefore for whole system examined one obtains a system of two Eqs. (11) and (12), with unknown boundary coefficients $p_{W}$ and $v_{W}$. Executing the substitution one can write the system of equations in the following form [3]

$$
\boldsymbol{H}_{R}\left(\boldsymbol{H}_{W}^{-1}-\boldsymbol{L}_{W} \boldsymbol{v}_{W}\right)-\boldsymbol{L}_{R} \boldsymbol{v}_{W}=-\boldsymbol{p}_{R} .
$$

After transforming and substituting $\boldsymbol{D}=\left(\boldsymbol{L}_{R}-\right.$ $\left.\boldsymbol{H}_{R} \boldsymbol{H}_{W}^{-1} \boldsymbol{L}_{W}\right)$ one obtains the following form of the solution:

$$
\boldsymbol{D} v_{W}=\boldsymbol{p}_{R} .
$$

The coefficients of the $\boldsymbol{D}$ matrix depend only on the room geometry and the analyzed sound frequency. According to Eq. (13) after performing the sufficient number of the acoustic pressure measurement inside the room one should be able to determine the surface parameter $v_{W}$ and determine the acoustic impedance $Z_{W}$ for the surfaces delimiting the examined room. If the matrix $\boldsymbol{D}^{-1}$, inverse to the $\boldsymbol{D}$ matrix, cannot be determined, then the exact solution does not exist and the approximate value of $v_{W}$ can be found by minimization of the mean-square error, i.e. $\min \left\|D v_{W}-p_{R}\right\|^{2}$.

The studies of the acoustic properties of the room have been carried out in a rectangular room, $6.7 \times 3.9 \times 2.85 \mathrm{~m}^{3}$ in size, normally utilized as a small workshop. Formulation of the problem how to determine the acoustic impedance of the walls of the room, assuming that the sound source is located on some vibrating part of a closed surface and that the acoustic pressure values within the room are known, by applying the inverse problem scheme, is presented in Fig. 3 .

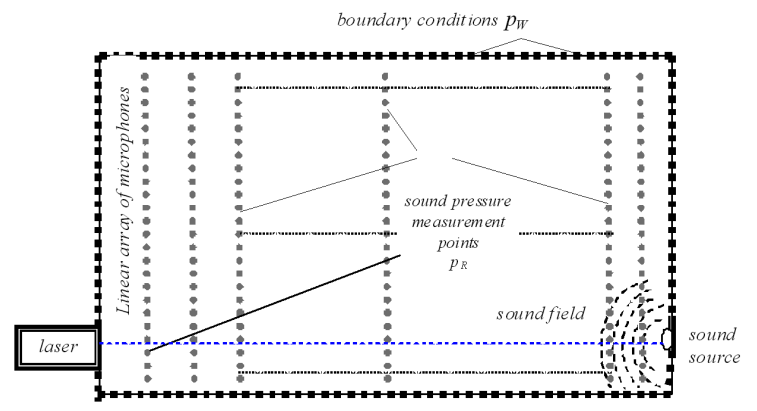

Fig. 3. Application of the inverse boundary element method in the interior acoustics problems - the test stand.

The first stage of the measurement has been carried out on a model of the actual room with a size reduction of 1:3 (Fig. 3). The walls of the model room have been made of OSB panels $2.2 \mathrm{~cm}$ thick. The density of the measurement grid, located at half height of the model room was one reception point per $5 \mathrm{~cm} \times 5 \mathrm{~cm}$ square. The sound source used for system excitation was a loudspeaker emitting sinusoidal signal, with laser monitored speed of the membrane vibration. The values of the acoustic pressure $p_{R}$ and acoustic signal phase shift have been simultaneously registered by 24 microphones located along a line in a perpendicular cross-section, at half-height of the lateral wall of the room. After measuring the acoustic pressure inside the model area, we can use formulae (11) and (12). All numerical operations have been carried out in the MATLAB environment. Figure 4 and Table present the results of the calculations.

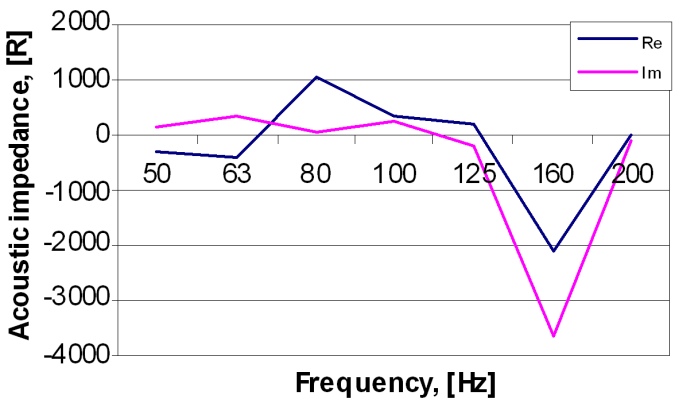

Fig. 4. Acoustic impedance $Z$ of the OSB panels.

TABLE

Acoustic impedance $Z$ of the OSB panels - numerical results.

\begin{tabular}{c|c|c}
\hline \hline$f[\mathrm{~Hz}]$ & $\operatorname{Re} Z$ & $\operatorname{Im} Z$ \\
\hline 50 & -310.6 & 126.3 \\
63 & -416.1 & 361.3 \\
80 & 1050.6 & 8.4 \\
100 & 357.1 & 274.4 \\
125 & 202.6 & -190.9 \\
160 & -2086 & -3656.1 \\
200 & -3.9 & -105
\end{tabular}

The calculation algorithm includes four main steps. The first step is the choice of points at which the acoustic pressures were measured during the experiment. The second is numerical procedures for determining the values of sound pressure at these points using the BEM calculations with the assumed acoustic impedance of the walls. Formulae (7)-(10) can be formulated as integral equations explicitly describing the considered boundary problem. The next step is to define the objective function as the sum of squared differences, and the last is optimization procedure which will search for the minimum objective function. After fulfilling the criterion of optimizing the calculations are finished. 


\section{Conclusion}

In this paper, algorithms of inversion methods have been shown to search for the acoustic characteristics of machines and acoustic properties of factory rooms. In both cases, the possibility of applying these methods in industrial environments were taken into account. The inverse method (in particular when applied with signal phase-shift measurement) is very sensitive both to the location of the substitute sound sources and distribution of observation points. This property can be also used for determination of positions of the real sound sources. The application of inverse methods in combination with other computer methods, like finite and boundary element methods, geometrical methods of acoustic signal propagation in enclosed spaces, leads to more effective ways of identification and localization of compound sound sources, as well as the determination of acoustic parameters of rooms. One of the main reasons for preferring the parameter estimation based on the inverse method is the fact that it can be practically applied for almost any geometry of the room. Experiments have shown that the inversion method can be applied to determine the acoustic parameters of machines in industrial conditions. We can use it to find the acoustic properties of wall materials for small industrial rooms. The presented method for determining the acoustic impedance $Z$ is constantly improved.

The equipment used for the study, in combination with appropriate software, allows a simultaneous, multi-channel registration of both acoustic pressure and phase shift of the registered acoustic signals, which is of considerable importance when the acquisition of huge data volumes has to be carried out.

\section{Acknowledgments}

This research work is supported by the Polish Ministry of Science and Higher Education grant no. N N 504342536

\section{References}

[1] Z. Engel, J. Piechowicz, L. Stryczniewicz, Fundamentals of Industrial Vibroacoustics, Ed. AGH, Kraków 2003 (in Polish).

[2] L. Stryczniewicz, J. Piechowicz, in: Proc. XXXV Winter School of Control Vibroacoustic Hazards, Gliwice-Ustron (Poland), Polish Acoustical Society, Gliwice 2007, p. 157 (in Polish).

[3] G.P. Nava, Y. Yasuda, S. Sakamoto, Acoust. Sci. Tech. 30, 100 (2009).

[4] J. Piechowicz, Int. J. Occupat. Safety Ergon. 17, 271 (2011).

[5] L. Stryczniewicz, J. Piechowicz, in: Proc. 2nd Int. Conf. on Experiments/Process/System Modelling/ Simulation and Optimization, Athens (Greece), Laboratory of Fluid Mechanics and Energy Patras, Athens 2007.

[6] R.D. Ciskowski, C.A. Brebbia, Boundary Element Methods in Acoustics, Computational Mechanics Publications and Elsevier Sci. Publ., Southampton 1991.

[7] A.A. Becker, The Boundary Element Method in Engineering. A Complete Course, McGraw-Hill, London 1992.

[8] J. Piechowicz, Arch. Acoust. 34, 169 (2009).

[9] Y. Kim, P.A. Nelson, J. Sound Vibrat. 275, 463 (2004).

[10] P.A. Nelson, S.H. Yoon, J. Sound Vibrat. 233, 643 (2000).

[11] A. Tarantola, Inverse Problem Theory, Elsevier Sci. B.V., Amsterdam 2002.

[12] H.W. Engl, M. Hanke, A. Neubauer, Regularisation of Inverse Problem, Kluver Academic Publisher, Boston 1996.

[13] J. Piechowicz, Arch. Acoust. 31, 287 (2006).

[14] A.N. Tikhonov, Dokl. Akad. Nauk SSR 153, 49 (1963) (in Russian).

[15] J. Piechowicz, Arch. Acoust. 32, 313 (2007). 